\section{LOCOMOTION}

\section{Stepping and Jumping}

IT is not really surprising that very few zoologists have investigated the gait of kangaroos but this situation has now been remedied by D. E. Windsor and A. I. Dagg of the University of Guelph in Canada. These authors have not only identified four types of gait in the Macropodinae, the marsupial subfamily which includes kangaroos and wallabies, but have also used this information to reconstruct the phylogeny of the group (J. Zool., 163, 165; 1971).

For their study, Windsor and Dagg took $360 \mathrm{~m}$ of film of nineteen species of macropod at seven zoos. Where possible, they placed stakes at measured intervals along each runway or they measured distances between fence supports so that they could calculate the speeds of the animals. This method was more accurate than measuring the locomotion of wild macropods in their natural habitats where the ground is often uneven and vegetation obscures movements. The four gaits identified were a slow progression, a walk, a quadrupedal bound and a bipedal hop.

The slow progression involves all the limbs plus the tail and is used chiefly while the animal is grazing. There were no significant differences between any of the species studied. This is to be expected, the authors say, because the centre of gravity of members of this group lies behind the hindfeet; thus the macropod has a triangle of support at the beginning and at the end of each stride and only during one part of the stride is the animal supported by two appendages alone.

The walk is the only gait in which the pairs of limbs are not used synchronously and is confined to the arboreal genus Dendrolagus, the tree kangaroo. This gait is similar to the common quadrupedal walk of most mammals. Without it, Dendrolagus would be restricted to branches which were sufficiently thick to permit the simultaneous use of the forelimbs or hindlimbs as in the slow progression.

The quadrupedal bound is considered to be a primitive gait because it is only found in the short tailed wallaby (Setonix brachyurus) and the tree kangaroos Dendrolagus matschiei and $D$. goodfellowi, which are believed to be close to the primitive phalangerid ancestors of macropods. This gait involves the use of the hindfeet and then the forefeet in sequence and it was found by Windsor and Dagg to be statistically similar in the three species which move in this way. Windsor and Dagg suggest that the ancestors of the present Macropodinae used this gait for movements faster than slow progression would allow. Because the ancestral habitat was rain forest, quad- rupedal gait would have allowed the primitive macropod to change direction as it dodged among the obstacles on the ground. The gait intergrades with the bipedal hop; it seems likely therefore that as rain forest gave way to open terrain and plains, some of the developing macropods took to using the faster bipedal locomotion and eventually the slower quadrupedal gait lost ground completely to the bipedal hop in the more highly developed plain dwelling species of Macropodinae. Faster gait was not necessarily a selective advantage in these large kangaroos because they evolved in an environment with few serious predators. The locomotory adaptation of large macropods in open terrain seems therefore to be for quick escape from danger rather than for maintaining high speeds as a means of outrunning predators.

Windsor and Dagg found that the pattern of bipedal hop varies considerably with the habitat of the species. Some use their hindfeet for a relatively long proportion of the stride, and some for a relatively short proportion of the stride. Species such as the wallaroo or euro, Macropus robustus, which live on rocky hills have increased suspension which allows them to mount steep slopes and leap among rocks. In contrast, the red kangaroo, Macropus rufus, which lives in open plains areas has a shorter period of suspension.

From their measurements, Windsor and Dagg draw several conclusions about the evolution of the Macropodinae and they have constructed a phylogenetic tree. The characteristics of the slow progression of the brush wallaby, Wallabia bicolour, suggest that it is closely related to the short tailed scrub wallaby (Setonix brachyurus), the New Guinean forest wallaby (Dorcopsis veterum), and the tree kangaroo Dendrolagus matschiei; but it is probably less primitive than Setonix and Dendrolagus because it does not seem to show quadrupedal bound. Windsor and Dagg support earlier work which favours removing all the species of Wallabia except $W$. bicolour to the genus Macropus, leaving the genus Wallabia as monotypic. Turning to Macropus, Windsor and Dagg say that the bipedal hop gait of the red kangaroo, $M$. rufus, is significantly different from that of $M$. robustus and $M$. melanops. This, they say, confirms previous suggestions (supported by chromosome counts) that $M$. rufus should be placed in a separate genus, Megaleia.

\title{
Water Abundance Weakens Apical Control
}

IN next Wednesday's Nature New Biology, fresh light is cast on the thorny botanical problem of how the apex of a growing plant controls the growth and development of the lateral buds farther down the stem. G. I. McIntyre of Regina Research Station, Saskatchewan, suggests that the supply of water to the lateral buds may play a much greater part in the phenomenon of apical dominance than had previously-been thought. His results indicate that when water is in short supply, the apex exerts complete control over the lateral buds and they show almost no growth. When water is freely available, however, this control is by no means so complete; when the relative turgidity of the leaves exceeds 80 per cent, the axillary buds are, to a large extent, released from apical control.

Apical dominance is the most obvious of the growth correlations shown by plants, those phenomena in which the growth of one region of the plant interacts with, controls or inhibits the growth of other parts of the plant. As such, apical dominance has been studied intensively, both for its own sake and in the hope of unearthing clues to the nature of the less overt correlation mechanisms. Nevertheless, the phenomenon has yielded few of its secrets.

Briefiy, extension growth in plants stems is confined chiefly to the apical regions of the main shoot. Lateral buds are formed at regular intervals down the main stem, but the further growth of these buds is inhibited; they are stimulated to develop further only if the shoot apex is destroyed, or if the control which the apex exerts over the laterals is broken in some other way.

Two principal explanations for this control are currently in vogue. The first holds that the buds are prevented from developing by a plant growth hormone or combination of plant growth hormones derived from the dominant apex and transmitted towards the base of the stem. The second theory is rather more controversial and is based on the idea of hormone-directed transport, a development of the nutrient diversion theory. This idea was formulated about thirty years ago and is based on the proved observation that nutrients flow preferentially towards regions of high hormone concentration. In other words, a more sophisticated version of the old "nutritive" theory, popular around the turn of the century.

McIntyre's theory could be interpreted as an extension of this latter idea, although he has no evidence to suggest what mechanism may direct the flow of water through the plant principally towards the dominant apex. But as he shows clearly that when water is in abundance, carbohydrate or nitrate availability is the limiting factor in breaking apical dominance, it is likely that some hormonal mechanism is responsible. 